\title{
ANÁLISIS DIMENSIONAL DEL RIESGO PERCIBIDO POR LA EXPOSICIÓN DEL PÚBLICO A RADIACIONES ELECTROMAGNÉTICAS EMITIDAS POR ESTACIONES BASE DE TELEFONÍA MÓVIL ${ }^{1}$
}

\author{
Lina María Gallego Serna ${ }^{2}$ \\ Javier Ignacio Torres Osorio ${ }^{3}$ \\ Jorge Abel Castañeda Salazar ${ }^{4}$
}

Recibido el 29 de junio de 2013 y aprobado el 27 de diciembre de 2013, actualizado el 9 junio de 2014

\section{RESUMEN}

La omnipresencia de estaciones base de telefonía móvil (EBTM) en los territorios urbanos ha dado lugar a que la población esté siendo expuesta a las radiaciones no ionizantes (RNI) emitidas por este tipo de fuentes radioeléctricas, dado que estas han sido ubicadas cerca de los sitios donde habitan y/o concurren las personas que se han considerado más susceptibles a las radiaciones electromagnéticas. Este escenario se ha caracterizado por las constantes preocupaciones de los ciudadanos y expertos en torno a los posibles efectos nocivos que se pueden presentar por la exposición a las $\mathrm{RNI}$; no obstante, existe también un ambiente de desconocimiento e incertidumbre frente al tema, lo que ha generado que sea más complejo evaluar este potencial riesgo de origen antrópico tecnológico. Por lo tanto, se consideró importante analizar la respuesta social frente al riesgo generado por las radiaciones electromagnéticas emitidas por EBTM, con el fin de realizar un diagnóstico integral de esta situación. Para ello, se adaptó el método del paradigma psicométrico, y se realizó un análisis descriptivo y multivariado, los cuales permitieron conocer la percepción social frente al riesgo en la zona que se consideró más crítica, según los resultados que surgieron del diagnóstico de las áreas urbanas expuestas a las RNI emitidas por las EBTM, localizadas en el municipio de Cartago (Valle del Cauca). Los resultados de los análisis muestran que la percepción de cada individuo se basa en juicios intuitivos y factores socioculturales, que se traducen en actitudes y decisiones que se toman frente al riesgo evaluado, que al parecer, en el imaginario social no ha sido concebido en su totalidad como un potencial factor de riesgo. Es por esto que se puede concluir que los juicios sobre el riesgo evaluado difieren no solo individualmente sino también colectivamente.

\section{PALABRAS CLAVE}

Percepción social del riesgo, radiaciones electromagnéticas no ionizantes, estaciones base de telefonía móvil, método del paradigma psicométrico. 


\title{
DIMENSIONAL ANALYSIS OF RISK PERCEIVED BECAUSE OF PUBLIC EXPOSURE TO ELECTROMAGNETIC RADIATIONS EMITTED BY MOBILE PHONE BASE STATIONS
}

\begin{abstract}
The omnipresence of mobile phone base stations (MPBS) in urban areas has resulted in the population being exposed to nonionizing radiation (NIR) emitted by this type of radio sources, inasmuch as they have been located near places inhabited or frequently visited by people who are considered more susceptible to electromagnetic radiations. This scenario has been characterized by the constant concerns of citizens and experts about the possible adverse health effects that may occur from exposure to NIR. However, there is also an atmosphere of ignorance and uncertainty regarding the topic which makes it more difficult to evaluate this technological anthropogenic potential risk.. Therefore, it was considered important to analyze the social response in front of the risk generated by the electromagnetic radiation emitted by MPBS, in order to make an integral diagnosis of this situation. To do this, the psychometric paradigm method was adapted and a descriptive and multivariate analysis was carried out, which allowed knowing the social perception of the risk in the area that was considered more critical according to the results that emerged from the diagnosis of urban areas exposed to NIR emitted by MPBS located in the municipality of Cartago (Valle del Cauca). The results of the analyses show that the perception of each individual is based on intuitive judgments and sociocultural factors which result in attitudes and decisions made against the assessed risk which, apparently, in the social imaginary has not been conceived in its entirety as a potential risk factor. That is why, it can be concluded that judgments about the assessed risk differ not only individually but also collectively.
\end{abstract}

\section{KEY WORDS}

Social perception of risk, non-ionizing electromagnetic radiations, mobile phone base stations, psychometric paradigm method.

\section{INTRODUCCIÓN}

La globalización de las telecomunicaciones y el desarrollo que ha tenido la industria de la telefonía móvil celular a nivel mundial, han posicionado al sector de las telecomunicaciones como un área estratégica para el desarrollo de las naciones, lo que se refleja en las políticas y planes de desarrollo del país (Visión Colombia Segundo Centenario 2019 y en el Plan Nacional de Desarrollo 2010-2014 "Prosperidad para todos"), y en la normatividad referente al tema (Ley 72 de 1989 y Decreto-Ley 1900 de 1990) ${ }^{6}$, las cuales promueven la utilización de las telecomunicaciones como instrumento para impulsar el desarrollo socioeconómico del país. Esto indica que los operadores de la telefonía móvil deben garantizar la cobertura y 
la calidad del servicio en todo el territorio colombiano, lo que ha dado lugar a la rápida expansión de fuentes emisoras de RNI (radiaciones no ionizantes) en las áreas urbanas y rurales del país, generándose así un problema ambiental, que ha sido denominado contaminación electromagnética no ionizante o "electropolución". Esta es un tipo de polución del aire, inmaterial y difícilmente perceptible, que hace referencia a la existencia de radiaciones electromagnéticas generadas de manera intencional por factores antrópicos, cuya exposición puede ocasionar efectos nocivos en el ambiente y en la salud de la población expuesta (v.g. síntomas neuroconductuales y cáncer) (Hyland, 2003; Torres y Alzate, 2006; Khurana et al., 2010; Gallego, 2011).

Este problema se ha acrecentado en la medida en que los operadores instalan mayor número de antenas y/o EBTM (estaciones base de telefonía móvil) en los municipios, para poder garantizar a los usuarios de la telefonía celular niveles óptimos de señal, los cuales llegaron a la cifra en Colombia de 49'066.359 de usuarios activos para el cuarto trimestre de 2012 (Ministerio de Tecnologías de la Información y las Comunicaciones -MinTIC-, 2013). Esto ha ocasionado un aumento en el número de personas expuestas a las radiaciones electromagnéticas generadas por las EBTM, que han sido ubicadas cerca de sitios que se han determinado como vulnerables (instituciones educativas, jardines infantiles, guarderías, hogares de bienestar familiar, centros de salud, hospitales, hogares geriátricos y centros penitenciarios). Este escenario, además, se ve agravado por la falta de directrices y restricciones en los Planes de Ordenamiento Territorial (POT) para la adecuada instalación de estas estaciones radioeléctricas en los territorios. Sin embargo, esto se ha debido principalmente a la incertidumbre científica y al desconocimiento que existe frente a los posibles efectos nocivos que se pueden derivar de la exposición a este tipo de fuentes radiantes, por ejemplo: afecciones del sistema circulatorio, trastornos del sueño, irritabilidad, depresión, dificultades de concentración, visión alterada, náuseas, pérdida del apetito, dolores de cabeza, fatiga o cansancio injustificado, malestar, vértigos, trastornos auditivos, pérdida de la memoria, mareos, alteraciones dérmicas, disminución de la libido o deseo sexual y, en el peor de los casos, cáncer (Santini et al., 2002; Navarro et al., 2003; Santini et al., 2003; Eger et al., 2004; Wolf y Wolf, 2004; Hutter et al., 2006; Abdel-Rassoul et al., 2007; Bortkiewicz et al., 2012).

Conforme con lo anterior, se puede indicar que este problema se ha convertido en el "protagonista" de un potencial escenario de riesgo de origen antrópico tecnológico, que a la luz de lo que plantea Lavell (1999), se caracteriza por tener dos factores interdependientes: la amenaza y la vulnerabilidad. El factor amenazante hace referencia a la probabilidad de que la exposición a las radiaciones electromagnéticas emitidas por las EBTM genere efectos biológicos adversos o impactos a la salud de la población que se encuentra cerca a estas fuentes radiantes; mientras que el factor vulnerable hace alusión a la susceptibilidad de las personas de ser afectadas por las RNI. No obstante, el estudio de este potencial escenario de riesgo no se puede reducir a un simple producto de probabilidades y 
consecuencias, sino que hay que tener en cuenta la percepción que el individuo tiene del riesgo; por lo tanto, es necesario que se analice el comportamiento de la población ante esta situación de riesgo, puesto que, al parecer, se ha visto como un problema individual y no como un problema social (colectivo).

Es por esto que, a partir del método del paradigma psicométrico, se realizó un análisis dimensional de la percepción del riesgo, para conocer la respuesta social al riesgo generado por las RNI emitidas por las EBTM, tomando como caso de estudio el barrio El Prado del municipio de Cartago (Valle del Cauca), que se identificó como una zona de alto riesgo, con base a los resultados del diagnóstico de las áreas urbanas expuestas a las $\mathrm{RNI}$ emitidas por las EBTM ${ }^{7}$.

\section{Percepción social del riesgo}

La percepción social del riesgo es un aspecto importante para evaluar el comportamiento de los individuos ante situaciones de riesgo; es decir que su análisis permite conocer la respuesta social frente a un riesgo evaluado. Sin embargo, hay que considerar que las valoraciones de los individuos y grupos difieren frente a un mismo hecho, dado que la percepción está condicionada por estructuras personales, cognoscitivas, emocionales y de motivación, así como por factores sociales, culturales, económicos, políticos y de familiaridad con la amenaza; por ende, la percepción incluye diferentes elementos que deben considerarse en conjunto para comprender cómo los individuos y los grupos sociales perciben los riesgos (Almaguer, 2008).

Según el paradigma psicométrico, se considera que la comprensión intuitiva del riesgo es un concepto multidimensional. En este sentido, las divergencias que se dan entre la percepción del público y la de los expertos no se deben solo a la ignorancia de las magnitudes del riesgo definidas por los especialistas en el tema, sino que existen otros elementos que las personas tienen en cuenta y que los expertos en ocasiones ignoran. Por consiguiente, se ha considerado que la población se compone de diferentes individuos, donde cada uno de ellos puede tener percepciones y marcos de referencia válidos y diferentes para un mismo riesgo (Portell y Solé, 2000; Vallejo y Vélez, 2009).

Es por esto que al momento de estudiar la percepción del riesgo se deben tomar en consideración cuestiones como: el conocimiento del riesgo (por parte de las personas expuestas y los expertos), el miedo, el control que supone el individuo que puede ejercer ante la amenaza, los efectos que pueden generar en la población, la posibilidad de ser impactado en lo personal, la confianza en las autoridades e instituciones encargadas de gestionar el riesgo, la gravedad de las consecuencias, la voluntariedad para realizar acciones preventivas, la demora de las consecuencias, el potencial catastrófico en un lapso corto y la magnitud del riesgo (Portell y Solé, 2000; Almaguer, 2008). 
Este tipo de aspectos o atributos son utilizados en el método del paradigma psicométrico; por ende, para analizar la percepción social del riesgo por la exposición a las RNI generadas por las EBTM se empleó este método, que tiene como objetivo obtener medidas cuantitativas del riesgo percibido y desarrollar taxonomías de factores de riesgo que permitan entender y predecir la respuesta social que originan. Además, este método permite identificar las similitudes y diferencias entre los grupos con respecto a las percepciones del riesgo (Portell y Solé, 2000).

En síntesis, el método del paradigma psicométrico es apropiado para entender la percepción social del riesgo, debido a que permite conocer cómo las personas comprenden las situaciones de riesgo a través de sus juicios, tomando en consideración las diferencias grupales (edad, género, nivel educativo de los encuestados, el tiempo de permanencia en el lugar, entre otras características), con el objetivo de explorar, además, la influencia de los factores sociales y culturales en las percepciones del riesgo (Portell y Solé, 2000; Almaguer, 2008).

\section{METODOLOGÍA}

La metodología empleada para analizar la percepción social del riego por exposición a las RNI emitidas por las EBTM, se basa en el método del paradigma psicométrico, el cual fue adaptado a las necesidades de la investigación, para poder cuantificar y modelar los factores que determinan las respuestas de los individuos frente a este potencial riesgo.

El diseño metodológico y los resultados que se exponen a continuación son producto del proyecto de grado denominado: Lineamientos para la gestión del riesgo de las áreas urbanas expuestas a las radiaciones electromagnéticas emitidas por las estaciones base de telefonía móvil. Estudio de caso: municipio de Cartago, Valle, de la Universidad Tecnológica de Pereira.

\section{Área de estudio}

Para analizar la percepción social del riesgo se tomó como área de estudio el barrio El Prado del municipio de Cartago (Valle del Cauca), que fue identificado como una zona de alto riesgo, al evaluar los factores amenazantes y vulnerables de los potenciales escenarios de riesgo del área urbana del municipio por exposición a las RNI emitidas por las EBTM. Cabe resaltar que los habitantes del área de estudio desconocían esta asignación, "zona de alto riesgo por exposición a RNI emitidas por EBTM", en el momento de realizar la encuesta para analizar la percepción social del riesgo.

El área de la EBTM ubicada en el barrio El Prado se caracteriza por tener más de dos estaciones bases ubicadas a menos de 300 metros una de la otra; además, se evidencian sitios vulnerables en las áreas de afectación de las RNI emitidas por la 
EBTM, que corresponden a un radio de 100, 200 y 300 metros con respecto a la fuente radioeléctrica ${ }^{8}$. Se encontró, en este caso, que una EBTM está ubicada a menos de 300 metros de dos centros de salud y dos jardines infantiles; situación que se hace más crítica en la medida en que algunos de estos sitios están localizados en el área de superposición de los CEM (campos electromagnéticos) generados por dos EBTM (ver Figura 1).

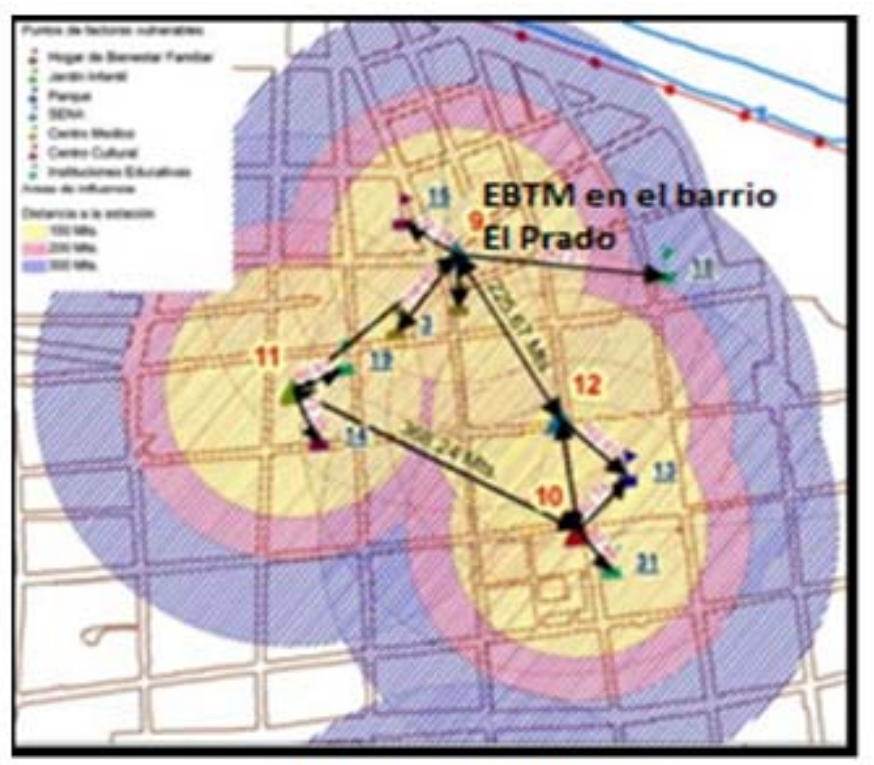

Fuente: Elaboración propia.

Figura 1. Area de alto riesgo por exposición a las RNI emitidas por la EBTM ubicada en el barrio EI Prado.

\section{Factor de riesgo a evaluar}

El factor de riesgo a evaluar se compuso de los posibles efectos nocivos que se pueden presentar en la salud de los individuos que se encuentran expuestos a las RNI emitidas por las EBTM.

\section{Selección de los atributos de riesgo a evaluar}

Los atributos de riesgo a evaluar fueron los nueve atributos establecidos en el documento Riesgo percibido: un procedimiento de evaluación de Portell y Solé (2000), así como la estimación de la magnitud del riesgo percibido (ver Tabla 1).

Según el paradigma psicométrico, la percepción del riesgo se basa en los juicios intuitivos; por ende, es necesario identificar cuáles son los atributos que expresan dicha percepción y que van a permitir entender la respuesta social del riesgo a evaluar. Por lo tanto, para el diseño y aplicación de la encuesta se tuvieron en cuenta estos nueve atributos que corresponden a: conocimiento por parte del sujeto expuesto, posible conocimiento por parte de los responsables de la gestión, temor que produce, vulnerabilidad, gravedad de las consecuencias, voluntariedad 
para realizar acciones preventivas, control, potencial catastrófico y tiempo de manifestación de los efectos o consecuencias (ver Tabla 1).

Tabla 1. Descripción de los atributos del riesgo evaluado en el método del paradigma psicométrico

\begin{tabular}{|c|c|}
\hline Atributos del riesgo & Descripción \\
\hline $\begin{array}{l}\text { A1. Conocimiento por parte } \\
\text { del sujeto expuesto }\end{array}$ & $\begin{array}{l}\text { Explora el conocimiento general que las personastienendelos } \\
\text { posibles daños que pueden desencadenarse del factor de } \\
\text { riesgo. }\end{array}$ \\
\hline $\begin{array}{l}\text { A2. Posible conocimiento por } \\
\text { parte de los responsables de } \\
\text { la gestión }\end{array}$ & $\begin{array}{l}\text { Indaga sobre el conocimiento general que las personas les } \\
\text { atribuyen a los responsables de la gestión en el municipio, con } \\
\text { respecto a los daños que pueden generarse del factor de } \\
\text { riesgo. El conocimiento de los responsables se ha relacionado } \\
\text { con la credibilidad y con las medidas de reducción o } \\
\text { prevención que se proponen o se llevan a cabo. }\end{array}$ \\
\hline A3. Temor que produce & $\begin{array}{l}\text { Evalúa la respuesta emocional de temor que representa para } \\
\text { las personas el factor de riesgo. }\end{array}$ \\
\hline A4. Vulnerabilidad & $\begin{array}{l}\text { Valora la percepción propia que se tiene de la susceptibilidad } \\
\text { de ser afectado por el factor de riesgo. }\end{array}$ \\
\hline $\begin{array}{l}\text { A5. Gravedad de las } \\
\text { consecuencias }\end{array}$ & $\begin{array}{l}\text { Explora la percepción de la gravedad de las consecuencias. La } \\
\text { gravedad se relaciona con la magnitud de la pérdida, que es } \\
\text { una de las variables que integran la definición de riesgo. }\end{array}$ \\
\hline $\begin{array}{l}\text { A6. Voluntariedad para realizar } \\
\text { acciones preventivas }\end{array}$ & $\begin{array}{l}\text { Está relacionada con la percepción de control del daño. } \\
\text { Pretende indagar la visión que tienen las personas con } \\
\text { respecto a su capacidad para realizar acciones preventivas. }\end{array}$ \\
\hline A7. Control & $\begin{array}{l}\text { Se centra en el grado de control percibido por las personas, } \\
\text { que frecuentemente está relacionado con la percepción de la } \\
\text { propia vulnerabilidad, lo que se ha denominado "optimismo } \\
\text { irrealista" (existe menos probabilidad de que yo me vea } \\
\text { afectado, porque no vivo al lado de la EBTM). } \\
\text { Frente a una intervención preventiva, el tema del control debe } \\
\text { abordarse con mucha cautela. Es necesario para descartar } \\
\text { actitudes fatalistas frente a una situación de riesgo (pasa } \\
\text { cuando pasa y yo no puedo evitar); sin embargo, una alta } \\
\text { percepción de control puede desencadenar un sentimiento de } \\
\text { invulnerabilidad. }\end{array}$ \\
\hline A8. Potencial catastrófico & $\begin{array}{l}\text { Explora el nivel de daño que puede generar el factor de riesgo } \\
\text { en un lapso corto. Este atributo mantiene una relación alta y } \\
\text { positiva con el riesgo total percibido. }\end{array}$ \\
\hline $\begin{array}{l}\text { A9. Demora de las } \\
\text { consecuencias }\end{array}$ & $\begin{array}{l}\text { Es un atributo que indaga sobre la percepción de la demora de } \\
\text { las consecuencias o de los impactos negativos. La demora es } \\
\text { un parámetro crítico en el momento de explicar las actitudes y } \\
\text { el comportamiento de las personas; en sí, cuando más } \\
\text { demoran las consecuencias menos nos impacta. Además, el } \\
\text { impacto no se percibe en un corto plazo ni se evidencia como } \\
\text { algo acumulativo en el tiempo. }\end{array}$ \\
\hline G1. Magnitud del riesgo & $\begin{array}{l}\text { Es un atributo de tipo global y se dirige a obtener una } \\
\text { estimación de la magnitud del riesgo percibido; en otras } \\
\text { palabras, la magnitud del riesgo es la medida que las personas } \\
\text { pueden atribuirle al riesgo, dicha medida es atribuida } \\
\text { subjetivamente por las personas. }\end{array}$ \\
\hline
\end{tabular}

Fuente: Bssado en Riesgo percibido: un procedimiento de evaluación (Portell y Solé, 2000).

\section{Estructura de la encuesta}

La encuesta diseñada para analizar la percepción social del riesgo en la zona seleccionada, está constituida por nueve preguntas que evalúan los atributos de riesgo (numeradas desde la A1 hasta la A9) y una pregunta global (G1) que pretende obtener una estimación de la magnitud del riesgo percibido.

Para cada pregunta de la encuesta se emplea una escala de 1 a 7. Los encuestados seleccionan el número que mejor represente su valoración (ver Tabla 2), por ejemplo: la posibilidad de que usted personalmente experimente un daño (inmediatamente o más adelante) como consecuencia del funcionamiento de las EBTM es: 
Tabla 2. Escala de estimación del atributo

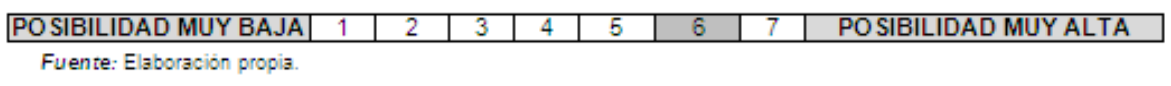

Con respecto a la pregunta sobre la magnitud del riesgo, se valora empleando una escala de 0 a 10 , siendo 0 riesgo muy bajo o nulo, y 10 riesgo alto o extremo.

Por otra parte, con el fin de conocer cómo las personas perciben el factor de riesgo con respecto a las diferencias grupales, se considera importante indagar sobre las características sociodemográficas de los encuestados, considerándose aspectos como: la edad, el género, el nivel de escolaridad y el tiempo que lleva habitando en la vivienda.

\section{Selección de la muestra}

Al tener formulada la encuesta, se procedió a determinar el tamaño de la muestra mediante un muestreo por selección sistemática, que dio como resultado una muestra de 71 viviendas para un total de 272 viviendas en un radio aproximado de 200 metros, con respecto a la estación base ubicada en el barrio El Prado del municipio de Cartago.

$$
\begin{aligned}
& n_{0}=\frac{z_{\alpha / 2}^{2} P Q}{e^{2}} n_{0}=\frac{(1.96)^{2}(0,5)(0,5)}{(0,1)^{2}} \approx 96 \\
& n=\frac{n_{0}}{1+\frac{h_{0}}{N}} \quad n=\frac{96}{1+\frac{96}{272}} \approx 71 \text { viviendas }
\end{aligned}
$$

\section{Método para el análisis de los resultados}

La encuesta fue aplicada a una muestra de 71 viviendas, que fue el equivalente a 71 habitantes mayores de 18 años que residen en el barrio El Prado del municipio de Cartago. Los resultados fueron analizados en Statistical Package for the Social Sciences (SPSS) versión 17.0. Para analizar los datos se realizaron dos tipos de análisis, un análisis descriptivo y un análisis de correspondencias múltiples (ACM). El análisis descriptivo consistió en caracterizar, describir y concluir sobre la muestra de los datos, lo que permitió conocer las respuestas más frecuentes para cada pregunta de la encuesta aplicada. Por el contrario, el análisis de correspondencias múltiples permitió relacionar las diferentes respuestas de las encuestas con las características sociodemográficas que se tuvieron presentes en el estudio (género, edad, nivel de escolaridad y tiempo que se lleva habitando en la vivienda), con lo cual se conocieron los patrones y las estructuras de relaciones que se dan entre las diferentes variables.

\section{Características sociodemográficas de los encuestados}

De los resultados obtenidos de las características sociodemográficas (género, edad, nivel de escolaridad y tiempo 
que se lleva habitando en la vivienda), se observó que de un total de 71 encuestados, el $63,4 \%$ fueron mujeres y el $36,6 \%$ fueron hombres. Con respecto a la edad, el $60,6 \%$ de los encuestados era mayores de 50 años, el 18,3\% se encontraba entre los 31 a 50 años y el 21,1\% estaba entre los 18 a 30 años. Según el nivel de escolaridad, el $46,5 \%$ de los encuestados había culminado sus estudios de bachillerato, en cambio, el $28,2 \%$ era profesional o se encontraba realizando sus estudios universitarios. Con relación al tiempo habitando la vivienda, se obtuvo como resultado final que el $47,9 \%$ de los encuestados llevaba más de 10 años, el 38,0\% solo llevaba entre 2 a 10 años y el 14,1\% llevaba menos de 2 años.

\section{RESULTADOS Y DISCUSIÓN}

\section{Análisis descriptivo}

Los resultados encontrados con respecto a cada atributo analizado se exponen a continuación.

Pregunta A1: ¿Qué tanto conoce usted sobre los posibles daños que puede causarle el funcionamiento de las EBTM?

Tabla 3. Resultados de la pregunta A1

\begin{tabular}{|l|c|c|c|c|}
\hline & Frecuencia & Porcentaje & Porcentaje válido & $\begin{array}{c}\text { Porcentaje } \\
\text { acumulado }\end{array}$ \\
\hline Nivel de conocimiento muy bajo (1) & 31 & 43,7 & 43,7 & 43,7 \\
Nivel de conocimiento bajo (2) & 5 & 7,0 & 7,0 & 50,7 \\
Nivel de conocimiento medio bajo (3) & 11 & 15,5 & 15,5 & 66,2 \\
Nivel de conocimiento medio (4) & 1 & 1,4 & 1,4 & 67,6 \\
Nivel de conocimiento medio alto (5) & 10 & 14,1 & 14,1 & 81,7 \\
Nivel de conocimiento alto (6) & 8 & 11,3 & 11,3 & 93,0 \\
Nivel de conocimiento muy alto (7) & 5 & 7,0 & 7,0 & 100,0 \\
Total & 71 & 100,0 & 100,0 & \\
\hline
\end{tabular}

Fuente: Elsborsción propis.

La mitad de los encuestados (50,7\%) manifestó tener un conocimiento muy bajo (valor 1) o bajo (valor 2) sobre los posibles daños que puede causar el funcionamiento de las EBTM (ver Tabla 3).

Pregunta A2: ¿Qué tanto considera usted que las entidades responsables de la prevención y la reducción del riesgo en el municipio conocen sobre los posibles daños generados por el funcionamiento de las EBTM? 
Tabla 4. Resultados de la pregunta A2

\begin{tabular}{|l|c|c|c|c|}
\hline & Frecuencia & Porcentaje & Porcentaje válido & $\begin{array}{c}\text { Porcentaje } \\
\text { acumulado }\end{array}$ \\
\hline Nivel de conocimiento muy bajo (1) & 16 & 22,5 & 22,5 & 22,5 \\
Nivel de conocimiento bajo (2) & 4 & 5,6 & 5,6 & 28,2 \\
Nivel de conocimiento medio bajo (3) & 11 & 15,5 & 15,5 & 43,7 \\
Nivel de conocimiento medio (4) & 6 & 8,5 & 8,5 & 52,1 \\
Nivel de conocimiento medio alto (5) & 8 & 11,3 & 11,3 & 63,4 \\
Nivel de conocimiento alto (6) & 6 & 8,5 & 8,5 & 71,8 \\
Nivel de conocimiento muy alto (7) & 20 & 28,2 & $\mathbf{2 8 , 2}$ & 100,0 \\
Total & 71 & 100,0 & 100,0 & \\
\hline
\end{tabular}

Fuente: Elaboración propis.

Del $52,1 \%$ de los encuestados, el $38,0 \%$ se destacó por considerar que las entidades responsables de la prevención y la reducción del riesgo en el municipio poseen un conocimiento muy bajo (valor 1: $22,5 \%$ ) y medio bajo (valor $3: 15,5 \%$ ) sobre los posibles daños generados por el funcionamiento de las EBTM. Por otra parte, el $28,2 \%$ del total de los encuestados (porcentaje mayor) consideró que las entidades tienen un nivel de conocimiento muy alto (valor 7 ) frente al tema (ver Tabla 4).

Las personas encuestadas que consideraron que las entidades responsables de la prevención y la reducción del riesgo en el municipio tienen conocimiento sobre los posibles daños generados por el funcionamiento de las antenas o EBTM, expresaron que a pesar de que cuentan con dicho conocimiento, las entidades del municipio no se pronuncian al respecto por pertenecer el servicio de telefonía móvil a empresas multinacionales. Por otra parte, las personas que señalaron que las entidades responsables de la prevención y la reducción del riesgo en el municipio conocen poco sobre los posibles daños generados por el funcionamiento de las EBTM, opinaron que si las entidades conocieran la situación ya se habrían pronunciado al respecto; además, consideraron que las personas a cargo no deben de saber nada sobre el tema.

Pregunta A3: ¿Cuánto le teme a los daños que puedan derivarse del funcionamiento de las EBTM?

Tabla 5. Resultados de la pregunta $A 3$

\begin{tabular}{|l|c|c|c|c|}
\hline & Frecuencia & Porcentaje & $\begin{array}{c}\text { Porcentaje } \\
\text { válido }\end{array}$ & $\begin{array}{c}\text { Porcentaje } \\
\text { acumulado }\end{array}$ \\
\hline Grado muy bajo (1) & 23 & 32,4 & 32,4 & 32,4 \\
Grado bajo (2) & 3 & 4,2 & 4,2 & 36,6 \\
Grado medio bajo (3) & 4 & 5,6 & 5,6 & 42,3 \\
Grado medio (4) & 7 & 9,9 & 9,9 & 52,1 \\
Grado medio alto (5) & 6 & 8,5 & 8,5 & 60,6 \\
Grado alto (6) & 3 & 4,2 & 4,2 & 64,8 \\
Grado muy alto (7) & $\mathbf{2 5}$ & 35,2 & 35,2 & 100,0 \\
Total & 71 & 100,0 & 100,0 & \\
\hline
\end{tabular}

Fuente: Elaboración propis. 
El 35,2\% de los encuestados manifestó sentir mucho temor frente a los posibles daños que pueden derivarse del funcionamiento de las EBTM; en comparación, el 32,4\% de los encuestados expresó sentir muy poco temor frente al caso (ver Tabla 5).

Los encuestados que manifestaron sentir un grado de temor muy alto frente a los daños que pueden derivarse del funcionamiento de las EBTM, se sustentaron, en gran parte, en el temor que sienten por el colapso de la estructura, primando esto frente a los daños que pueden presentarse en la salud a causa de la exposición a las RNI emitidas por las EBTM. Por el contrario, los encuestados que señalaron sentir un grado de temor muy bajo, lo dijeron principalmente por el poco conocimiento que tienen sobre los posibles daños que pueden generarse por la exposición a las radiaciones electromagnéticas que son emitidas por estas fuentes radiantes.

Pregunta A4: La posibilidad de que Ud. personalmente experimente un daño (inmediatamente o más adelante) como consecuencia del funcionamiento de las EBTM es:

Tabla 6. Resultados de la pregunta A4

\begin{tabular}{|l|c|c|c|c|}
\hline & Frecuencia & Porcentaje & $\begin{array}{c}\text { Porcentaje } \\
\text { válido }\end{array}$ & $\begin{array}{c}\text { Porcentaje } \\
\text { acumulado }\end{array}$ \\
\hline Posibilidad muy baja (1) & 11 & 15,5 & 15,5 & 15,5 \\
Posibilidad baja (2) & 5 & 7,0 & 7,0 & 22,5 \\
Posibilidad medio baja (3) & 7 & 9,9 & 9,9 & 32,4 \\
Posibilidad media (4) & 9 & 12,7 & 12,7 & 45,1 \\
Posibilidad medio alta (5) & 19 & 26,8 & 26,8 & 71,8 \\
Posibilidad alta (6) & 8 & 11,3 & 11,3 & 83,1 \\
Posibilidad muy alta (7) & 12 & 16,9 & 16,9 & 100,0 \\
Total & 71 & 100,0 & 100,0 & \\
\hline
\end{tabular}

Fuente: Elsboración propis.

El $71,8 \%$ de los encuestados se caracterizó, en gran parte, por considerar que tiene una posibilidad medio alta (valor 5: 26,8\%: porcentaje mayor) y muy baja (valor 1: 15,5\%) de experimentar un daño como consecuencia del funcionamiento de las EBTM; mientras que, un 16,9\% del total de los encuestados manifestó que la posibilidad es muy alta (valor 7) (ver Tabla 6).

El $43,7 \%$ de los encuestados que manifestó tener una probabilidad medio alta $(26,8 \%)$ o muy alta $(16,9 \%)$ de ser afectado por las radiaciones electromagnéticas emitidas por las EBTM, se considera susceptible o vulnerable a los daños que pueden presentarse en la salud, por habitar cerca de una EBTM. Por otro lado, las personas que expresaron que la probabilidad de ser afectados a causa del funcionamiento de las EBTM es muy baja (15,5\%), lo hicieron, en gran medida, por considerar que existe menos probabilidad de ser afectados, dado que su vivienda no se encuentra al lado de la estación base. 
Pregunta A5: En caso de que el funcionamiento de las EBTM genere una afectación en la salud, ¿qué tan grave considera usted que pueden ser los daños?

Tabla 7. Resultados de la pregunta A5

\begin{tabular}{|l|c|c|c|c|}
\hline & Frecuencia & Porcentaje & $\begin{array}{c}\text { Porcentaje } \\
\text { válido }\end{array}$ & $\begin{array}{c}\text { Porcentaje } \\
\text { acumulado }\end{array}$ \\
\hline Gravedad muy baja (1) & 2 & 2,8 & 2,8 & 2,8 \\
Gravedad baja (2) & 3 & 4,2 & 4,2 & 7,0 \\
Gravedad medio baja (3) & 6 & 8,5 & 8,5 & 15,5 \\
Gravedad media (4) & 11 & 15,5 & 15,5 & 31,0 \\
Gravedad medio alta (5) & 14 & 19,7 & 19,7 & 50,7 \\
Gravedad alta (6) & 12 & 16,9 & 16,9 & 67,6 \\
Gravedad muy alta (7) & $\mathbf{2 3}$ & $\mathbf{3 2 , 4}$ & $\mathbf{3 2 , 4}$ & $\mathbf{1 0 0 , 0}$ \\
Total & $\mathbf{7 1}$ & $\mathbf{1 0 0 , 0}$ & $\mathbf{1 0 0 , 0}$ & \\
\hline
\end{tabular}

Fuente: Elaboración propis.

El $52,1 \%$ de los encuestados opinó que en el caso de que las radiaciones electromagnéticas emitidas por las EBTM generen una afectación en la salud, la gravedad de los daños es medio alta $(19,7 \%)$ y muy alta $(32,4 \%)$; lo que permite concluir que cualquier afectación a la salud por más leve que sea, se considera como un daño muy grave, puesto que se ve afectada la calidad de vida de las personas expuestas (ver Tabla 7).

Pregunta A6: ¿En qué grado puede evitar que el funcionamiento de las EBTM afecte su entorno?

Tabla 8. Resultados de la pregunta A6

\begin{tabular}{|l|c|c|c|c|}
\hline & Frecuencia & Porcentaje & $\begin{array}{c}\text { Porcentaje } \\
\text { válido }\end{array}$ & $\begin{array}{c}\text { Porcentaje } \\
\text { acumulado }\end{array}$ \\
\hline Grado muy bajo (1) & 34 & 47,9 & 47,9 & 47,9 \\
Grado bajo (2) & 6 & 8,5 & 8,5 & 56,3 \\
Grado medio bajo (3) & 9 & 12,7 & 12,7 & 69,0 \\
Grado medio (4) & 5 & 7,0 & 7,0 & 76,1 \\
Grado medio alto (5) & 7 & 9,9 & 9,9 & 85,9 \\
Grado alto (6) & 4 & 5,6 & 5,6 & 91,5 \\
Grado muy alto (7) & 6 & 8,5 & 8,5 & 100,0 \\
Total & 71 & 100,0 & 100,0 & \\
\hline
\end{tabular}

Fuente: Elaboración propis.

El 47,9\% de los encuestados señaló que puede evitar en un grado muy bajo que el funcionamiento de las EBTM afecte su entorno (ver Tabla 8); sin embargo, algunas de estas personas expresaron que no habían hecho nada frente al caso, puesto que el lote donde está instalada la estación base pertenece a una persona de gran poder en el municipio de Cartago y en el Norte del Valle del Cauca, razón por la cual sienten temor al manifestar sus objeciones con respecto a la localización de la estación base. Además, otra parte de los encuestados desconoce los derechos que tiene como ciudadanos; es decir, que no conoce los debidos procesos para presentar sus objeciones frente a la 
otorgación de la licencia o el permiso para la instalación de la infraestructura de telefonía móvil en un determinado lugar. Por otra parte, los encuestados que expresaron que pueden evitar que el funcionamiento de las EBTM afecte su entorno, lo hicieron porque consideran que existen dos medidas: irse del barrio (situación que todavía no está contemplada) o mandar un derecho de petición a la entidad competente.

Pregunta A7: ¿Qué posibilidad considera usted que tiene para reducir los daños que puede causarle el funcionamiento de las EBTM?

Tabla 9. Resultados de la pregunta A7

\begin{tabular}{|l|c|c|c|c|}
\hline & Frecuencia & Porcentaje & $\begin{array}{c}\text { Porcentaje } \\
\text { válido }\end{array}$ & $\begin{array}{c}\text { Porcentaje } \\
\text { acumulado }\end{array}$ \\
\hline Posibilidad de control muy baja (1) & 44 & 62,0 & 62,0 & 62,0 \\
Posibilidad de control baja (2) & 2 & 2,8 & 2,8 & 64,8 \\
Posibilidad de control medio baja (3) & 8 & 11,3 & 11,3 & 76,1 \\
Posibilidad de control medio (4) & 4 & 5,6 & 5,6 & 81,7 \\
Posibilidad de control medio alto (5) & 8 & 11,3 & 11,3 & 93,0 \\
Posibilidad de control alto (6) & 2 & 2,8 & 2,8 & 95,8 \\
Posibilidad de control muy alto (7) & 3 & 4,2 & 4,2 & 100,0 \\
Total & 71 & 100,0 & 100,0 & \\
\hline
\end{tabular}

Fuente: Elsborsción propis.

El 62,0\% de los encuestados que manifestó tener una posibilidad de control muy baja para reducir los daños que puede causarle el funcionamiento de las EBTM (ver Tabla 9), expresó que no conoce las medidas adecuadas para ser menos susceptibles a las RNI emitidas por estas fuentes radiantes.

Pregunta A8: ¿Qué tanto el funcionamiento de las EBTM puede afectar a las personas en un lapso de 2 a 5 años?

Tabla 10. Resultados de la pregunta A8

\begin{tabular}{|l|c|c|c|c|}
\hline & Frecuencia & Porcentaje & $\begin{array}{c}\text { Porcentaje } \\
\text { válido }\end{array}$ & Porcentaje acumulado \\
\hline Grado muy bajo (1) & 5 & 7,0 & 7,0 & 7,0 \\
Grado bajo (2) & 3 & 4,2 & 4,2 & 11,3 \\
Grado medio bajo (3) & 3 & 4,2 & 4,2 & 15,5 \\
Grado medio (4) & 17 & 23,9 & 23,9 & 39,4 \\
Grado medio alto (5) & 15 & 21,1 & 21,1 & 60,6 \\
Grado alto (6) & 14 & 19,7 & 19,7 & 80,3 \\
Grado muy alto (7) & 14 & 19,7 & 19,7 & 100,0 \\
Total & 71 & 100,0 & 100,0 & \\
\hline
\end{tabular}

Fuente: Elaboración propis.

El $84,4 \%$ de los encuestados consideró que el grado de afectación generado por la exposición a las radiaciones electromagnéticas emitidas por las EBTM, en un lapso de 2 a 5 años, puede ser medio (23,9\%), medio alto $(21,1 \%)$, alto $(19,7 \%)$ y muy alto $(19,7 \%)$ (ver Tabla 10); no obstante, las personas que manifestaron un grado de afectación medio, lo hicieron porque reconocen que las afectaciones a la salud por el funcionamiento 
de las EBTM son más evidentes y pueden presentarse en un lapso superior al establecido en la pregunta.

Pregunta A9: ¿Cuándo considera usted que se manifiestan los efectos generados por el funcionamiento de las EBTM?

Tabla 11. Resultados de la pregunta A9

\begin{tabular}{|l|c|c|c|c|}
\hline & Frecuencia & Porcentaje & $\begin{array}{c}\text { Porcentaje } \\
\text { válido }\end{array}$ & $\begin{array}{c}\text { Porcentaje } \\
\text { acumulado }\end{array}$ \\
\hline De forma inmediata (1-2) & 1 & 1,4 & 1,4 & 1,4 \\
En un corto plazo (3-4) & 7 & 9,9 & 9,9 & 11,3 \\
En un largo plazo (5-7) & 63 & 88,7 & 88,7 & 100,0 \\
Total & 71 & 100,0 & 100,0 & \\
\hline
\end{tabular}

Fuente: Elaboración propia.

El 88,7\% (63 encuestados) consideró que los efectos generados por el funcionamiento de las EBTM se manifiestan en un largo plazo (ver Tabla 11).

Pregunta G1: ¿Cómo valora el riesgo que puede generar en la salud el funcionamiento de las EBTM?

Tabla 12. Resultados de la pregunta G1

\begin{tabular}{|l|c|c|c|c|}
\hline & Frecuencia & Porcentaje & $\begin{array}{c}\text { Porcentaje } \\
\text { válido }\end{array}$ & $\begin{array}{c}\text { Porcentaje } \\
\text { acumulado }\end{array}$ \\
\hline Riesgo muy bajo (0) & 5 & 7,0 & 7,0 & 7,0 \\
Riesgo bajo (1-2) & 4 & 5,6 & 5,6 & 12,7 \\
Riesgo medio bajo (3-4) & 5 & 7,0 & 7,0 & 19,7 \\
Riesgo medio (5) & 8 & 11,3 & 11,3 & 31,0 \\
Riesgo medio alto (6-7) & 12 & 16,9 & 16,9 & 47,9 \\
Riesgo alto (8-9) & 11 & 15,5 & 15,5 & 63,4 \\
Riesgo muy alto (10) & 26 & 36,6 & 36,6 & 100,0 \\
Total & 71 & 100,0 & 100,0 & \\
\hline
\end{tabular}

Fuente: Elaboración propis.

El 36,6\% (porcentaje mayor) consideró que la magnitud del riesgo generado por el funcionamiento de las EBTM es muy alto; mientras que el $32,4 \%$ de los encuestados se caracterizó por considerar que el riesgo es medio alto (valores entre 6 y 7 ) y alto (valores 8 y 9) (ver Tabla 12).

Se puede decir que la mitad de los encuestados desconoce los posibles efectos que ocasionan las RNI emitidas por las EBTM en la salud de las personas expuestas; no obstante, estos mismos encuestados reconocen que son vulnerables frente a esta amenaza, que sus efectos se presentan en un largo plazo y que su capacidad de prevención y control es muy baja; esto generó que se estimara la magnitud del riesgo como muy alta. 


\section{Análisis de Correspondencias Múltiples (ACM)}

Las respuestas de las preguntas A1 (conocimiento por parte del sujeto expuesto), A2 (posible conocimiento por parte de los responsables de la gestión), A3 (temor que produce) y A9 (demora de las consecuencias) se caracterizaron porque estuvieron relacionadas principalmente con dos aspectos sociodemográficos: el nivel de escolaridad y el tiempo que lleva habitando en la vivienda. Con respecto a las preguntas A4 (vulnerabilidad) y A5 (gravedad de las consecuencias), la variable que mejor se correlacionó fue el nivel de escolaridad; mientras que, en las preguntas A6 (voluntariedad para realizar acciones preventivas) y A7 (control), las respuestas estuvieron relacionadas especialmente con el género y la edad. Por otra parte, las preguntas A8 (potencial catastrófico) y G1 (magnitud del riesgo) se relacionaron con todas las variables (género, edad, nivel de escolaridad y tiempo que lleva habitando en la vivienda).

Lo anterior, permite concluir que las respuestas de cada pregunta de la encuesta variaron según los grupos que se formaron con relación a estas características sociodemográficas; lo que demuestra que la percepción del riesgo está influenciada por las diferentes características sociodemográficas de las personas. Sin embargo, los resultados que arrojó el análisis de correspondencias múltiples no muestran una diferencia tan marcada entre los grupos y sus diferentes respuestas, lo que se puede deber a que no se tiene tanto conocimiento de los alcances de este factor de riesgo, puesto que no es considerado como un riesgo tan común, como lo son los riesgos por fenómenos naturales. Por lo tanto, es normal encontrar casos en los que mujeres con estudios técnicos o universitarios manifestaran tener un conocimiento alto sobre los posibles daños que se presentan por la exposición a las RNI emitidas por las EBTM; como a las vez, se presentaron casos en los que las mujeres con este nivel de estudio expresaron saber poco sobre el tema.

\section{CONCLUSIONES}

Es esencial que la percepción social del riesgo se emplee como instrumento para el desarrollo de políticas de prevención y mitigación del riesgo, y que se incluya en los estudios de los escenarios de riesgo, dado que permite realizar un diagnóstico integral del riesgo evaluado y hace factible que se establezcan estrategias de gestión de la problemática. Esto se da, puesto que las respuestas obtenidas en el análisis descriptivo y en el análisis de correspondencias múltiples dan a entender que no se debe evaluar a la población como un todo, debido a que se pone de manifiesto las diferencias de percepciones y las prioridades de los individuos.

La falta de medidas de precaución frente a los posibles efectos perjudiciales para la salud por la exposición a las radiaciones electromagnéticas emitidas por las EBTM, ha generado un 
potencial escenario de riesgo, el cual no ha sido reconocido en su totalidad por las autoridades locales y miembros de la comunidad, pues parecería que solo puede ser considerado como riesgo cuando los efectos a la salud por la exposición a las $\mathrm{RNI}$ estén comprobados científicamente, para lo cual pueden pasar muchos años.

Es necesario que la comunidad sea informada y conozca sobre los alcances o los impactos que se derivan de la exposición a las RNI emitidas por las EBTM, dado que el desconocimiento con respecto a los efectos nocivos que se pueden derivar de la exposición a estas radiaciones electromagnéticas, hace a la comunidad más vulnerable a la amenaza y a que se quebranten sus derechos; asimismo, el desconocimiento de estos derechos y de los mecanismos de participación ciudadana que se deben emplear en dichos casos, agravan más la situación, generando que no se ejerza una presión social.

Es importante que se investigue la forma en que los medios de comunicación influyen en la percepción social del riesgo, puesto que generalmente el conocimiento frente a los posibles efectos nocivos en la salud por la exposición a las RNI emitidas por las EBTM, se adquiere en mayor parte de dichos medios; por lo tanto, la percepción del riesgo y los grados de vulnerabilidad tienden a variar, teniendo en cuenta la información que se transmite; además, se debe estudiar cómo cambia el imaginario social con respecto al vecindario y ciudad cuando se instala una EBTM.

\section{REFERENCIAS}

- Abdel-Rassoul, G., El-Fatech, O.A., Salem, M.A., Michael, A., Farahat, F., El-Batanouny, M. y Salem, E. (2007). Neurobehavioral effects among inhabitants around mobile phone base stations. Neurotoxicology, 28(2), 434-440.

- Almaguer, C. (2008). El riesgo de desastre: una reflexión filosófica (Tesis de Doctorado). Universidad de La Habana. Cuba. Recuperado de http://www.eumed.net/tesis/ 2009/cdar/Los\%20estudios\%20sobre\%20percepcion\%20soci al\%20del\% 20riesgo\%20el\%20enfoque\%20psicometrico.htm

- Bortkiewicz, A., Gadzicka, E., Szyjkowska, A., Politanski, P., Mamrot, P., Szymczak, W. y Zmyslony, M. (2012). Subjective complaints of people living near mobile phone base stations in Poland. Int J Occup Med Environ Health, 25(1), 31-40.

- Colombia. Congreso de la República. (1989). Ley 72 (20, diciembre, 1989). Diario Oficial No. 39.111.

- Colombia. Ministerio de Comunicaciones. (1990). Decreto-Ley 1900 (19, Agosto, 1990). Diario Oficial No. 39.507.

- Departamento Nacional de Planeación. (2010). Plan Nacional de Desarrollo 2010-2014 "Prosperidad para todos". Bogotá: Autor.

- Eger, H., Hagen, K.U., Lucas, B., Vogel, P. y Voit, H. (2004). Einfluss der aumlichennahe von mobilfunksendeanlagen auf die krebsinzidenz (Influence of the spatial proximity of mobile phone 
base stations on cancer rates). Umwelt-Medizin-Gesellschaft, 17, 273-356.

- Gallego, L. (2011). Lineamientos para la gestión del riesgo de las áreas urbanas expuestas a las radiaciones electromagnéticas emitidas por las estaciones base de telefonía móvil. Estudio de caso: municipio de Cartago, Valle (Tesis de pregrado laureada). Universidad Tecnológica de Pereira, Pereira. Recuperado de http://repositorio.utp.edu.co/xml/handle/

11059/53/browse? value=Gallego+Serna\%2C+Lina+Mar\%C3\% ADa\&type=author

- Hutter, H.P., Moshammer, H., Wallner, P. y Kundi, M. (2006). Subjective symptoms, sleeping problems, and cognitive performance in subjects living near mobile phone base stations. Occup. Environ. Med., 63, 307-313.

- Hyland, G. (2003). ¿Me oyes? ¿No? ¿Y ahora? Contaminación electromagnética. El uso de teléfono móvil. Guía de Salud Laboral 21.2 Recuperado de http://www.cgtbbva.net/derechos/salud_laboral/cuadernos/gs I21.pdf

- Khurana, V., Hardell, L., Everaert, J., Bortkiewicz, A., Carlberg, M. y Ahonen, M. (2010). Epidemiological evidence for a health risk from mobile phone base stations. Int J Occup Environ Health, 16(3), 263-267.

- Lavell, A. (1999). Gestión de Riesgos Ambientales Urbanos. Facultad Latinoamericana de Ciencias Sociales (FLACSO) y La Red de Estudios Sociales en Prevención de Desastres en América Latina (LA RED). Recuperado de http://www.desenredando.org/public/articulos/1999/grau/ GestionDeRiesgosAmbientalesUrbanos-1.0-sep-12-2001.pdf

- Ministerio de Tecnologías de la Información y las Comunicaciones -MinTIC-. (2013). Boletín Trimestral de las TIC. Cifras cuarto trimestre de 2012. Bogotá: Autor.

- Navarro, E.A., Segura, J., Portolés, M. y Gómez-Perretta de Mateo, C. (2003). The Microwave Syndrome: A Preliminary Study in Spain. Rev. Electromagnetic Biology and Medicine, 22, 161169.

- Portell, M. y Solé, M. (2000). NTP 578: Riesgo percibido: un procedimiento de evaluación. Ministerio de Trabajo y Asuntos Sociales, España. Recuperado de http://www.insht.es/InshtWeb/Contenidos/Documentacion/Fic hasTecnicasl NTP/Ficheros/501a600/ntp_578.pdf

- Presidencia de la República de Colombia y Departamento Nacional de Planeación. (2005). Visión Colombia Segundo Centenario: 2019. Resumen Ejecutivo. Recuperado de https://www.dnp.gov.co/Portals/0/archivos/documentos/2019/ Libro_4ta_ed/ resumen_ejecutivo.pdf

- Santini, R., Santini, P., Danze, J.M., Le Ruz, P. y Seigne, M. (2002). Study of the Health of People Living in the Vicinity of Mobile Phone Base Stations: I. Influences of Distance and Sex. Rev. Pathol Biol, 50, 369-373.

- (2003). Symptoms experienced by people in vicinity of base stations: II/ Incidences of age, duration of exposure, location of subjects in relation to the antennas and other electromagnetic factors. Rev. Pathol Biol, 51, 412-415. 
- Torres, J. y Alzate, L. (2006). Efectos de las radiaciones electromagnéticas no ionizantes en sistemas biológicos. Revista Médica de Risaralda, 12(2), 44-54.

- Vallejo, A. y Vélez, J. (2009). La percepción del riesgo en los procesos de urbanización del territorio. Revista Letras Verdes, 3, 29-31.

- Wolf, R. y Wolf, D. (2004). Increased Incidence of Cancer Near a Cell-Phone Transmitter Station. Int J Cancer Prev., 1, 123-128.

1. Este artículo es un resultado parcial del proyecto titulado: "Definición de una metodología para la realización de estudios en áreas con alto riesgo de exposición a las radiaciones electromagnéticas generadas por estaciones base de telefonía móvil; caso de estudio Manizales, Caldas" Código 0457011, y continuación del proyecto de grado denominado: Lineamientos para la gestión del riesgo de las áreas urbanas expuestas a las radiaciones electromagnéticas emitidas por las estaciones base de telefonía móvil. Estudio de caso: municipio de Cartago, Valle. Trabajo de grado laureado por la Universidad Tecnológica de Pereira.

2. Administradora Ambiental. Integrante del Grupo de Investigación Campos Electromagnéticos, Medio Ambiente y Salud Pública, Universidad de Caldas. Grupo de Investigación en Gestión Ambiental Territorial, Universidad Tecnológica de Pereira. nali_g@hotmail.com

3. M.Sc. Instrumentación Física. Integrante del Grupo de Investigación Campos Electromagnéticos, Medio Ambiente y Salud Pública, Universidad de Caldas. javier.torres@ucaldas.edu.co

4. M.Sc. Instrumentación Física. Universidad de Caldas. jorge.castaneda_s@ucaldas.edu.co

5. En el documento Visión Colombia Segundo Centenario: 2019. Resumen Ejecutivo, se establece que el sector de las telecomunicaciones deberá "contribuir a consolidar una sociedad informada, conectada e integrada al entorno global, para lo cual deberá incorporar continuamente los últimos desarrollos tecnológicos, particularmente adecuarse a la convergencia de redes, terminales y servicios. Además, el sector deberá propender por una cobertura adecuada y acceso universal para toda la población". Por otra parte, en el Plan Nacional de Desarrollo 2010-2014 se define que: "el Gobierno nacional, a través del Ministerio de TIC, fomentará el despliegue y uso eficiente de la infraestructura para la provisión de redes de telecomunicaciones y los servicios que sobre ellas se puedan prestar, y promoverá el óptimo aprovechamiento de los recursos escasos con el ánimo de generar competencia, calidad y eficiencia, en beneficio de los usuarios. Se buscará la expansión, y cobertura para zonas de difícil acceso, en especial beneficiando a poblaciones vulnerables [...]".

6. La Ley 72 de 1989 establece que el Gobierno Nacional promoverá la cobertura nacional de los servicios de telecomunicaciones y su modernización, a fin de propiciar el desarrollo socioeconómico de la población. De la misma forma, el Decreto-Ley 1900 de 1990 establece que las telecomunicaciones deberán ser utilizadas como instrumentos para impulsar el 
desarrollo político, económico y social del país, con el objeto de elevar el nivel y la calidad de vida de los habitantes.

7. Los resultados del diagnóstico de las áreas urbanas expuestas a las RNI emitidas por las EBTM, se pueden observar en el documento: Lineamientos para la gestión del riesgo de las áreas urbanas expuestas a las radiaciones electromagnéticas emitidas por las estaciones base de telefonía móvil. Estudio de caso: municipio de Cartago, Valle, cuya referencia se encuentra en el presente artículo. Además, los resultados se exponen en el artículo: "Metodología para el diagnóstico de áreas urbanas con alta exposición a radiaciones electromagnéticas emitidas por estaciones base de telefonía móvil", el cual fue aceptado para ser publicado en esta Revista.

8. Se establecieron estas tres áreas específicas como zonas de afectación de las RNI emitidas por las EBTM, puesto que estudios epidemiológicos como los de Santini et al. (2002), Navarro et al. (2003), Santini et al. (2003), Eger et al. (2004) y Bortkiewicz et al. (2012) han relacionado algunos efectos perjudiciales en la salud humana o patologías específicas con cada una de estas áreas aledañas a las EBTM.

Para citar este artículo: Gallego-Serna, L.M., Torres-Osorio, J.I., Castañeda-Salazar, J.A. (2014). Análisis dimensional del riesgo percibido por la exposición del público a radiaciones electromagnéticas emitidas por estaciones base de telefonía móvil.

Revista Luna Azul, 39, 105-123. Recuperado de http://lunazul.ucaldas.edu.colindex.php?option=content\&tas k=view\&id $=950$ 